\title{
SUR LA CORRESPONDANCE PAR PARALLELISME DE DEUX SURFACES
}

\author{
P. DRĂGILǍ
}

\section{INTRODUCTION}

1. Il est bien connu que Peterson fut le premier qui a étudié la correspondance de deux surfaces, par parallélisme de leurs normales, aux points homologues. Il croyait avoir démontré qu'on peur choisir le même système de coordonnées curvilignes $u, v$ sur les deux surfaces $S, \bar{S}$, de manière que les tangentes aux lignes $u, v$ de la première surface $S$ soient parallèle avec les tangentes correspondantes aux lignes $u, v$ de la deuxième surface $\bar{S}$, ce que signifie que les paramètres directeurs de ces tangentes vérifient les relations

$$
\frac{\bar{x}_{u}}{x_{u}}=\frac{\bar{y}_{u}}{y_{u}}=\frac{\bar{z}_{u}}{z_{u}}=\lambda ; \quad \frac{\bar{x}_{v}}{x_{v}}=\frac{\bar{y}_{v}}{y_{v}}=\frac{\bar{z}_{v}}{z_{v}}=\mu,
$$

$\lambda$ et $\mu$ étant fonctions de deux variables $u, v$. Il a énoncé ensuite dans son mémoire [2, pp. 5-6] le théoreme IV:

Si deux surfaces $x, y, z$ et $X, Y, Z$ se trouvait dans une relation telle que les normales aux points correspondantes soient parallèles, alors par chaque point d'une surface passent deux directions qui sont paralleles (ou deux courbes dont les tangentes sont parallèles) aux directions correspondantes sur l'autre surface. Ces deux directions, sur chaque surface, sont conjuguées et par conséquent, constituent sur les deux surfaces les réseaux auxquels nous avons donné le nom de base de la relation.

Il a répété l'énonce de ce théoreme encore une fois [2, p. 26]:

Si deux surfaces $x, y, z$ et $X, Y, Z$ se trouvent dans la relation de parallélisme, par chaque point de l'une des surfaces passent deux courbes qui sont parallèle (c'est-a-dire qui ont des tangentes parallèles) aux courbes correspondantes sur la surface $X, Y, Z$. Sur les deux surfaces ces courbes sont conjuguées et constituent, par conséquent, la base de la relation. Nous les appellerons courbes de base.

L'énonce du théoreme de Peterson est fausse à deux points de vue. D'abord c'est inexact que si deux surfaces se trouvent dans la relation de parallélisme, par chaque point de l'une des surfaces passent deux courbes qui sont paralleles aux courbes correspondantes sur l'autre surface. Nous montrerons ainsi qu'y a-t-il des couples de deux surfaces, en relation de parallélisme, sur lesquelles il n'y a pas de systèmes de courbes parallèles. En second lieu, c'est inexact que les deux direc-

Received by the editors April 26, 1957. 
tions sur la première surface, qui sont parallèles aux directions correspondantes sur l'autre surface, sont toujours conjuguées sur les deux surfaces. Nous montrerons, dans ce qui suit, que, en dehors du cas des couples de deux surfaces étudiées par Peterson, qui sont caractérisées par les équations (1), il y a encore des couples de surfaces dont les plans tangents aux points homologues sont parallèles et qui sont définies par un autre système d'équations, dans quel cas, au moins sur l'une de deux surfaces, les deux directions ne sont plus conjuguées.

2. C'est une chose curieuse que la même faute se répète encore chez d'autres géomètres, même chez les uns des plus grands, par exemple Guichard. Il est très probable qu'il n'a pas été influencé directement par les recherches antérieures de Peterson, qui furent d'abord publiées en langue russe. En étudiant les réseaux conjugués dans l'espace $S_{n}$, il croit avoir aussi démontré que les réseaux sont les seuls systèmes parallèles. Nous citons d'après $[3$, pp. 5-6]:

Soient $x_{1}, x_{2}, \cdots, x_{n}, x_{1}^{\prime}, x_{2}^{\prime}, \cdots, x_{n}^{\prime}, 2 n$ fonctions de deux variables définissant les coordonnées de deux points $M, M^{\prime}$. On dit que ces deux points décrivent des systèmes parallèles si l'on a les relations

$$
\frac{\partial x_{i}^{\prime}}{\partial u}=\alpha \frac{\partial x_{i}}{\partial u}, \quad \frac{\partial x_{i}^{\prime}}{\partial v}=\beta \frac{\partial x_{i}}{\partial v} .
$$

La condition de compatibilité montre que les quantités $x$ sont solutions de l'équation de Laplace

$$
\frac{\partial^{2} x}{\partial u \partial v}=-\frac{\frac{\partial \alpha}{\partial v}}{\alpha-\beta} \cdot \frac{\partial x}{\partial u}+\frac{\frac{\partial \beta}{\partial u}}{\alpha-\beta} \cdot \frac{\partial x}{\partial v} .
$$

Il résulte de la que les réseaux sont les seuls systèmes qui admettent des parallèles.

I.es recherches de Peterson sur le parallélisme des surfaces et sur quelques problèmes de nature projective furent continuées par plusieurs géomètres, parmi lesquels les roumains et les russes sont les plus nombreux. Malheureusement une partie de leurs conclusions sont aussi fausse. Nous nous bornerons actuellement de mentionner seulement ceux qui se sont occupés de la correspondance par parallélisme de deux surfaces. Dans les mémoires [4] de M. Haimovici et I. Popa et puis dans [5] de $\mathrm{A}$. Myller se répète le raisonemment et la démonstration fausse de Peterson. Nous citons d'après [5, p. 12]:

12. Considerînd perechile de puncte depe douà suprafete $S_{1}$ si $S_{2}$. 
unde planele tangente sînt paralele, se stabileşte o corespondentă între punctele celor doü̆ suprafete, care a fost numită corespondentă prin plane paralele.

Unei curbe $C_{1}$ pe $S_{1}$ î corespunde o curbă $C_{2}$ pe $S_{2}$. Ne punem întrebarea cînd aceste curbe strîmbe sînt paralele. ...

Ele formează o retea.

Retelele de curbe paralele sînt conjugate.

Systemes de SURFACES PARALlÈles SANS RÉSEAUX PARALlèleS

3. Un des plus curieux cas, peut-être, c'est celui des couples de deux surfaces parallèles, sur lesquelles il n'y a pas des courbes correspondantes parallèles.

(Nous désignons dans nos calculs, comme d'abitude, les dérivées $\left.\partial r / \partial u, \partial r / \partial v, \partial^{2} r / \partial u^{2}, \partial^{2} r / \partial u \partial v \cdots \operatorname{par} r_{u}, r_{v}, r_{u}^{2}, r_{u v}, \cdots.\right)$

Nous pouvons chercher les surfaces $S, \bar{S}$ de ce genre, en écrivant les relations de condition

$$
\frac{\bar{r}_{u}}{r_{v}+a y_{u}}=\lambda, \quad \frac{\hat{r}_{v}}{r_{v}+b r_{u}}=\mu,
$$

c'est-a-dire

$$
\begin{aligned}
& \frac{\bar{x}_{u}}{x_{v}+a x_{u}}=\frac{\bar{y}_{u}}{y_{v}+a y_{u}}=\frac{\bar{z}_{u}}{z_{v}+a z_{u}}=\lambda, \\
& \frac{\bar{x}_{v}}{x_{v}+b x_{u}}=\frac{\bar{y}_{v}}{y_{v}+b y_{u}}=\frac{\bar{z}_{u}}{x_{v}+b z_{u}}=\mu .
\end{aligned}
$$

Nous nous bornerons actuellement a traiter seulement le cas le plus simple:

$$
\begin{array}{lll}
\bar{x}_{u}=x_{v}+x_{u}, & \bar{y}_{u}=y_{v}+y_{u}, & \bar{z}_{u}=z_{v}+z_{u}, \\
\bar{x}_{v}=x_{v}-x_{u}, & \bar{y}_{v}=y_{v}-y_{u}, & \bar{z}_{v}=z_{v}-z_{u},
\end{array}
$$

quand les expressions des coordonnées $x, y, z, \bar{x}, \bar{y}, \bar{z}$ sont des fonctions harmoniques de deux variables $u, v$. Cherchons maintenant a faire un changement de variables

$$
u=\phi(s, t), \quad v=\psi(s, t)
$$

de manière que les directions nouvelles des tangentes $r_{s}, r_{t}$, respectivements $\bar{r}_{s}, \bar{r}_{l}$, sur les deux surfaces $S, \bar{S}$, soient parallèles, ce que signifie qu'elles doivent satisfaire les relations

$$
\frac{\bar{x}_{s}}{x_{s}}=\frac{\bar{y}_{s}}{y_{s}}=\frac{\bar{z}_{s}}{z_{s}}, \quad \frac{\bar{x}_{t}}{x_{t}}=\frac{\bar{y}_{t}}{y_{t}}=\frac{\bar{z}_{t}}{z_{t}} .
$$


Considerons ensuite le couple de deux surfaces

$$
\left\{\begin{array} { l } 
{ x = u + 2 v , } \\
{ y = y ( u , v ) } \\
{ z = z ( u , v ) }
\end{array} \quad \left\{\begin{array}{l}
\bar{x}=3 u+v \\
\bar{y}=\int\left(y_{v}+y_{u}\right) d u+\left(y_{v}-y_{u}\right) d v \\
\bar{z}=\int\left(z_{v}+z_{u}\right) d u+\left(z_{v}-z_{u}\right) d v
\end{array}\right.\right.
$$

$y(u, v), z(u, v)$ étant deux fonctions harmoniques, pour lesquelles sont satisfaites les relations (2). Calculons les nouvelles dérivées $x_{s}, x_{t}, \cdots, \bar{x}_{s}, \bar{x}_{t}, \cdots$ et en faisant les substitutions dans les relations (3) nous obtenons

$$
\begin{aligned}
& \frac{3 u_{s}+v_{s}}{u_{s}+2 v_{s}}=\frac{\left(y_{v}+y_{u}\right) u_{s}+\left(y_{v}-y_{u}\right) v_{s}}{y_{u} u_{s}+y_{v} v_{s}}=\frac{\left(z_{v}+z_{u}\right) u_{s}+\left(z_{v}-z_{u}\right) v_{s}}{z_{u} u_{s}+z_{v} v_{s}}=\lambda^{x}, \\
& \frac{3 u_{t}+v_{t}}{u_{t}+2 v_{t}}=\frac{\left(y_{v}+y_{u}\right) u_{t}+\left(y_{v}-y_{u}\right) v_{t}}{y_{u} u_{t}+y_{v} v_{t}}=\frac{\left(z_{v}+z_{u}\right) u_{t}+\left(z_{v}-z_{u}\right) v_{t}}{z_{u} u_{t}+z_{v} v_{t}}=\mu^{x},
\end{aligned}
$$

et, après avoir effectué les calculs et les réductions nécessaires, on constate que les deux égalités qui en résultent

$$
\begin{aligned}
& \left(2 y_{u}-y_{v}\right)\left(u_{s}^{2}+v_{s}^{2}\right)=0, \\
& \left(2 y_{u}-y_{v}\right)\left(u_{t}^{2}+v_{t}^{2}\right)=0
\end{aligned}
$$

sont impossibles. Nous avons ainsi démontré qu'il y en a des couples de surfaces, dans la relation de parallélisme, sur lesquelles il n'y a pas de réseaux réelles parallèles.

\section{Nouveaux RÉSEAUX DE PARALlÉLISME}

4. En dehors du cas de parallélisme des systèmes de deux directions conjuguées sur les deux surfaces $S, \bar{S}$ étudié par Peterson, Guichard et ses continuateurs, il y a encore un autre cas, quand les tangentes aux lignes $u$ de la première surface sont parallèles avec les tangentes aux lignes $v$ de la deuxième surface, et les tangentes aux lignes $v$ de la première surface sont parallèles avec les tangentes aux lignes $u$ de la deuxième surface. Nous supposons, bien entendu, que nous avons choisi le même système de coordonnées curvilignes $u, v$ sur les deux surfaces. Les paramètres directeurs des tangentes vérifient alors le système d'équations

$$
\frac{\bar{x}_{u}}{x_{v}}=\frac{\bar{y}_{u}}{y_{v}}=\frac{\bar{z}_{u}}{z_{v}}=\lambda, \quad \frac{\bar{x}_{v}}{x_{u}}=\frac{\bar{y}_{v}}{y_{u}}=\frac{\bar{z}_{v}}{z_{u}}=\mu,
$$


c'est-a-dire

$$
\frac{\bar{r}_{u}}{r_{v}}=\lambda, \quad \frac{\bar{r}_{v}}{r_{v}}=\mu,
$$

$\lambda$ et $\mu$ étant deux fonctions arbitraires mais continues et dérivables de $u, v$. Nous pouvons maintenant calculer les paramètres directeurs de la normale a la surface $S$

$$
\left|\begin{array}{ll}
x_{u} & y_{u} \\
x_{v} & y_{v}
\end{array}\right|, \quad\left|\begin{array}{ll}
y_{u} & z_{u} \\
y_{v} & z_{v}
\end{array}\right|, \quad\left|\begin{array}{cc}
z_{u} & x_{u} \\
z_{v} & x_{v}
\end{array}\right|,
$$

et puis a la surface $\bar{S}$

$$
\left|\begin{array}{ll}
\bar{x}_{u} & \bar{y}_{u} \\
\bar{x}_{v} & \bar{y}_{v}
\end{array}\right|=\lambda \mu\left|\begin{array}{ll}
x_{u} & y_{u} \\
x_{v} & y_{v}
\end{array}\right|, \cdots
$$

ce qu'on peut écrire encore

$$
\left|\begin{array}{l}
\bar{r}_{u} \\
\bar{r}_{v}
\end{array}\right|=\lambda \mu\left|\begin{array}{l}
r_{u} \\
r_{v}
\end{array}\right|
$$

Il est aisé de voir que les plans tangents, aux points homologues des deux surfaces $S$ et $\bar{S}$, sont parallèles.

5. Entre ces deux cas de relation de parallélisme des surfaces il y a des différences profondes de nature géométrique intrinseque, lesquelles ne sont pas suffisamment visibles dans les équations de conditions (4), $\left(4^{\prime}\right)$. Pour cette considération il est nécessaire de donner l'interpretation géométrique correspondante.

Dans le premier cas les tangentes menées à une courbe de la première surface $S$, dans les points $M_{1}, M_{2}, M_{3}$ sont parallèles avec les tangentes, menées aux points homologues $M_{1}^{\prime}, M_{2}^{\prime}, M_{3}^{\prime}$, à la courbe correspondante de la deuxième surface.

C'est le cas de correspondance par parallélisme de Peterson-Guichard, que nous l'appellerons correspondance par parallélisme linéaire; les courbes correspondantes sur les deux surfaces constituent un système de réseaux parallèles.

Dans le second cas, au contraire, les tangentes menées à une courbe de la première surface $S$, dans les points $M_{1}, M_{2}, M_{3}$ sont parallèles avec les tangentes, menées aux points homologues $M_{1}^{\prime}, M_{2}^{\prime}, M_{3}^{\prime}$, sur la deuxième surface $\bar{S}$, aux courbes différentes.

Ce nouveau cas de correspondance nous l'appellerons correspondance par parallélisme ponctuel; les courbes sur les deux surfaces constituent alors un nouveau système de réseaux que nous les appellerons réseaux quasi-parallèles. 
6. Maintenant il suit de montrer qu'il y en a des couples de deux surfaces, qui satisfassent nos conditions de parallélisme et qui vérifient alors les relations (4), $\left(4^{\prime}\right)$. A cet effet supposons que la première surface $S$ est rapportée aux lignes asymptotiques, ce que signifie que les expressions de ses coordonnées satisfont au système d'équations

$$
\begin{aligned}
& r_{u u}=a^{\prime} r_{u}+b^{\prime} r_{v}, \\
& r_{u v}=a r_{u}+b r_{v}+c r, \\
& r_{v v}=a^{\prime \prime} r_{u}+b^{\prime \prime} r_{v}
\end{aligned}
$$

(pour lesquelles sont remplies les conditions d'intégrabilité).

Du système $\left(4^{\prime}\right)$ on obtient

$$
\bar{r}_{u}=\lambda r_{v}, \quad \bar{r}_{v}=\mu r_{u},
$$

et, en posant ensuite la condition d'intégrabilité

$$
\left(\lambda r_{v}\right)_{v}=\left(\mu r_{u}\right)_{u}
$$

il résulte

$$
\lambda\left(a^{\prime \prime} r_{u}+b^{\prime \prime} r_{v}\right)+\lambda_{v} r_{v}-\mu\left(a^{\prime} r_{u}+b^{\prime} r_{v}\right)-\mu_{u} r_{u}=0 .
$$

Pour que cette relation (6) soit satisfaite par les expressions des trois coordonnées, il faut et il suffit que les coefficients de deux dérivées $r_{u}, r_{v}$ soient nuls, c'est-a-dire:

$$
\lambda a^{\prime \prime}-\mu a^{\prime}-\mu_{u}=0, \quad \lambda b^{\prime \prime}+\lambda_{v}-\mu b^{\prime}=0 .
$$

Il est manifeste que, quelque soient les coefficients $a^{\prime}, b^{\prime}, a^{\prime \prime}, b^{\prime \prime}$, il est toujours possible de trouver deux fonctions $\lambda(u, v), \mu(u, v)$, qui satisfont les relations (7).

Supposant que ces relations de conditions (7) soient satisfaites, nous étudierons actuellement le réseau de la deuxieme surface $\bar{S}$, dont les directions des tangentes aux lignes $u$ et $v$ sont parallèles aux directions asymptotiques de la première surface $S$. Pour cela écrivons les deux dérivées

$$
r_{v}=\frac{\bar{r}_{u}}{\lambda}, \quad r_{u}=\frac{\bar{r}_{v}}{\mu},
$$

puis calculons les dérivées du second ordes

$$
\begin{aligned}
& r_{v v}=\frac{\bar{r}_{u v}}{\lambda}-\frac{\lambda_{v} \bar{r}_{u}}{\lambda^{2}}, \\
& r_{u u}=\frac{\bar{r}_{u v}}{\mu}-\frac{\mu_{u} \bar{r}_{v}}{\mu^{2}},
\end{aligned}
$$


et, en les substituant ensuite dans la première et la troisième équation du système (5), nous obtenons

$$
\begin{aligned}
& \bar{r}_{u v}=\frac{b^{\prime} \mu}{\lambda} \bar{r}_{u}+\left(a^{\prime}+\frac{\mu_{u}}{\mu}\right) \bar{r}_{v}, \\
& \bar{r}_{u v}=\left(b^{\prime \prime}+\frac{\lambda_{v}}{\lambda}\right) \bar{r}_{u}+\frac{a^{\prime \prime} \lambda}{\mu} \bar{r}_{v} .
\end{aligned}
$$

Ces deux equations (8) sont équivalentes en cas que les relations (7) sont satisfaites. Il est aisé de voir que les lignes $u$, $v$ sur la deuxième surface $\bar{S}$ forment alors un réseau conjugué, que nous l'appellerons réseau conjoint du première réseau, formé par les lignes asymptotiques de la première surface $S$. Nous avons établi ainsi une nouvelle correspondance entre deux surfaces, que nous désignerons correspondance $P_{0}$. Nous pouvons énoncer ensuite le théoreme suivant:

Si les courbes $u, v$ sont des asymptotiques sur une surface $S$, alors les deux familles des courbes conjointes $u, v$, sur la deuxième surface $\bar{S}$, dont les directions des tangentes sont respectivement parallèles aux directions asymptotiques de la première surface $S$, forment un réseau conjugué.

7. Nous pouvons maintenant aller un peu plus loin. Considérons une surface $S$, dont les expressions des coordonées soient trois solutions linéairement indépendantes du système

$$
\begin{aligned}
& r_{u u}=a^{\prime} r_{u}+b^{\prime} r_{v}+c^{\prime} r, \\
& r_{u v}=a r_{u}+b r_{v}+c r, \\
& r_{v v}=a^{\prime \prime} r_{u}+b^{\prime \prime} r_{v},
\end{aligned}
$$

ce que signifie que les lignes $v$ sont des asymptotiques pour cette surface. En substituant l'expression de la derivée $r_{v v}$ dans l'equation

$$
\lambda r_{v v}+\lambda_{v} r_{v}-\mu r_{u u}-\mu_{u} r_{u}=0
$$

il vient

$$
r_{u u}=\frac{\lambda a^{\prime \prime}-\mu_{u}}{\mu} r_{u}+\frac{\lambda b^{\prime \prime}+\lambda_{v}}{\mu} r_{v},
$$

ce que signifie que les lignes $u$ constituent le deuxième système des asymptotiques sur la surface $S$. Nous savons déjà que dans ce cas le réseau conjoint de l'autre surface $\bar{S}$ est conjugué.

Maintenant il suit de considérer le cas le plus général, quand les expressions des coordonnées d'une surface $S$ sont trois solutions 
linéairement indépendantes d'un système

$$
\begin{aligned}
& r_{u v}=a r_{u}+b r_{v}+c r, \\
& r_{u u}=a^{\prime} r_{u}+b^{\prime} r_{v}+c^{\prime} r, \\
& r_{v v}=a^{\prime \prime} r_{u}+b^{\prime \prime} r_{v}+c^{\prime \prime} r .
\end{aligned}
$$

Calculons les dérivées

$$
\begin{aligned}
& r_{u u}=\frac{\bar{r}_{u v}}{\mu}-\frac{\mu_{u} \bar{r}_{v}}{\mu^{2}}, \\
& r_{u v}=\frac{\bar{r}_{u v}}{\mu}-\frac{\mu_{v} \bar{r}_{v}}{\mu^{2}}, \\
& r_{v v}=\frac{\bar{r}_{u v}}{\lambda}-\frac{\lambda_{v} \bar{r}_{u}}{\lambda^{2}},
\end{aligned}
$$

et, en faisant ensuite les substitutions dans ces équations des derivées $r_{u u}, r_{u v}, r_{v v}$, nous voyons que les expressions des coordonnées de la seconde surface $\bar{S}$ vérifient un système d'équations de la même forme que (10), ce que signifie que les lignes $u, v$, sur la surface $\bar{S}$, ne sont plus conjuguées, ni asymptotiques.

Nous avons ainsi établit une correspondance par parallélisme plus général, que nous appellerons correspondance $P_{0}$ Dans un cas particulier quand les courbes $u, v$ sont asymptotiques, respectivement lignes conjuguées, nous parlerons des réseaux $P_{0}$ asymptotiques, respectivement des réseaux $P_{0}$ conjugués.

Quelques familles Des SuRfaces aux RÉseaux $P_{0}$ ASymptotiques

8. Nous allons étudier, dans ce qui suit, quelques familles des surfaces rapportées aux lignes asymptotiques, et chercher ensuite les familles des réseaux, dont les directions de deux tangentes soient parallèles aux directions correspondantes des asymptotiques de la première famille des surfaces.

Considérons d'abord les surfaces simplement réglées, qui sont définies par le système d'équations

$$
\begin{aligned}
& r_{u u}=a^{\prime} r_{u}+b^{\prime} r_{v}, \\
& r_{u v}=a r_{u}+b r_{v}+c r, \\
& r_{v v}=b^{\prime \prime} r_{v} .
\end{aligned}
$$

Calculons les deux dérivées $r_{u u}, r_{v v}$ des relations $\left(4^{\prime}\right)$ et en les substituant ensuite dans (11) nous obtenons 


$$
\begin{aligned}
& \bar{r}_{u v}=\frac{b^{\prime} \mu}{\lambda} \bar{r}_{u}+\left(a^{\prime}+\frac{\mu_{u}}{\mu}\right) \bar{r}_{v}, \\
& \bar{r}_{u v}=\left(b^{\prime \prime}+\frac{\lambda_{v}}{\lambda}\right) \bar{r}_{u} .
\end{aligned}
$$

En considérant que dans ce cas de même sont satisfaites les relations (7) il est aisé de voir que les deux équations (12) sont équivalentes, ayant le coefficient de la dérivée $\bar{r}_{v}$ nul; dans ce cas l'invariant $k$ est aussi nul. On peut démontrer aussi, que les directions des tangentes aux lignes conjuguées, définies par une équation de Laplace dont l'un de deux coefficients des dérivées $\bar{r}_{u}, \bar{r}_{v}$ est nul, sont parallèles avec les directions asymptotiques d'une surface simplement reglées.

EXEMPLE. Les directions asymptotiques de la surface réglée $S$

$$
x=e^{u+v}, \quad y=e^{2 u+v}, \quad z=e^{3 u}
$$

sont parallèles aux directions conjuguées de la surface $\bar{S}$

$$
\bar{x}=e^{v-2 u}, \quad \bar{y}=2 e^{v-u}, \quad \bar{z}=3 v,
$$

qui n'est pas réglée.

9. Considérons les surfaces doublement réglées, qui sont définies par les équations

$$
\begin{aligned}
r_{u u} & =a^{\prime} r_{u}, \\
r_{u v} & =a r_{u}+b r_{v}+c r, \\
r_{v v} & =b^{\prime \prime} r_{v} .
\end{aligned}
$$

En continuant le même raisonnement et en effectuant le même calcul que dans le cas précédent, nous obtenons deux équations

$$
\begin{aligned}
& \bar{r}_{u v}=\left(a^{\prime}+\frac{\mu_{u}}{\mu}\right) \bar{r}_{v}, \\
& \bar{r}_{u v}=\left(b^{\prime \prime}+\frac{\lambda_{v}}{\lambda}\right) \bar{r}_{u}
\end{aligned}
$$

qui sont simultanément satisfaites pour

$$
a^{\prime}+\frac{\mu_{u}}{\mu}=0, \quad b^{\prime \prime}+\frac{\lambda_{v}}{\lambda}=0 .
$$

Nous sommes arrivés ainsi au résultat suivant: Les directions asymptotiques d'une surface doublement réglée sont respectivement 
parallèles aux directions conjuguées d'un réseau défini par l'équation de Laplace

$$
\bar{r}_{u v}=0 .
$$

On peut démontrer aisément aussi le théoreme réciproque que les directions des tangentes d'un réseau (14) sont parallèles avec les directions asymptotiques d'une surface doublement réglée.

Exemples. Les directions asymptotiques du paraboloïde $S$

$$
x=u, \quad y=v, \quad z=u v
$$

sont parallèles respectivement aux directions conjuguées de la surface $\bar{S}$

$$
\bar{x}=\frac{v^{m}}{m}, \quad \bar{y}=\frac{u^{n}}{n}, \quad \bar{z}=\frac{u^{n+1}}{n+1}+\frac{v^{m+1}}{m+1},
$$

( $m$ et $n$ étant deux constantes réelles arbitraires), qui en général n'est plus réglée.

Les directions asymptotiques du hiperboloïde $S$

$$
x=\frac{u+v}{1+u v}, \quad y=\frac{v-u}{1+u v}, \quad z=\frac{u v-1}{1+u v}
$$

sont parallèles aux directions conjuguées de la surface $\bar{S}$

$$
\bar{x}=u-\frac{u^{3}}{3}+v-\frac{v^{3}}{3}, \quad \bar{y}=u+\frac{u^{3}}{3}-v-\frac{v^{3}}{3}, \quad \bar{z}=u^{2}+v^{2} .
$$

Nous avons ainsi prouvé que les surfaces réglées peuvent être en relation de parallélisme avec des surfaces non-réglées, contrairement a l'affirmation de Peterson [2, pp. 27-28].

10. Considérons ensuite les surfaces Tzitzeica, caractérisées par la propriété que l'invariant $K / d^{4}$, ou $K$ est la courbure en un point $M$ de la surface, et $d$ la distance de l'origine au plan tangent au point $M$ à la surface, soit constant. Ces surfaces sont définies par le système d'équations

$$
\begin{aligned}
& \boldsymbol{r}_{u u}=\frac{h_{u}}{h} \boldsymbol{r}_{u}+\frac{1}{h} \boldsymbol{r}_{0}, \\
& \boldsymbol{r}_{u v}=h \boldsymbol{r}, \\
& \boldsymbol{r}_{v v}=\frac{1}{h} \boldsymbol{r}_{u}+\frac{h_{v}}{h} \boldsymbol{r}_{v},
\end{aligned}
$$


$h$ étant une solution particulière de l'équation

$$
(L h)_{u v}=h-\frac{1}{h^{2}} .
$$

En suivant le même procédé que dans les cas antérieurs, nous obtenons les deux équations

$$
\begin{aligned}
& \bar{r}_{u v}=\frac{\mu}{\lambda h} \bar{r}_{u}+\left(\frac{h_{u}}{h}+\frac{\mu_{u}}{\mu}\right) \bar{r}_{v}, \\
& \bar{r}_{v u}=\left(\frac{h_{u}}{h}+\frac{\lambda_{v}}{\lambda}\right) \bar{r}_{u}+\frac{\lambda}{\mu h} \bar{r}_{v},
\end{aligned}
$$

qui sont aussi équivalentes.

EXEMPLE. En prenant

$$
h=1
$$

nous trouvons la surface $S$

$$
x=e^{m_{1}\left(u+m_{1} v\right)}, \quad y=e^{m_{2}\left(u+m_{2} v\right)}, \quad z=e^{m_{3}\left(u+m_{3} v\right)},
$$

( $m_{i}$ étant les trois racines cubiques de l'unité), dont les directions asymptotiques sont parallèles aux directions conjuguées de la surface $\bar{S}$

$$
\bar{x}=\gamma_{1} e^{\alpha_{1} u+\beta_{1} v}, \quad \bar{y}=\gamma_{2} e^{\alpha_{2} u+\beta_{2} v}, \quad \bar{z}=\gamma_{3} e^{\alpha_{3} u+\beta_{3} v},
$$

$\alpha_{i}, \beta_{i}, \gamma_{i}$ étant les constantes

$$
\begin{aligned}
\alpha_{i} & =1+m_{i}, \\
\beta_{i} & =1+m_{i}^{2}, \\
\gamma_{i} & =\frac{m_{i}}{1+m_{i}^{2}},
\end{aligned}
$$

avec $\lambda=\mu=e^{u+v}$. Les coordonnées de cette dernière surface vérifient l'équation de Laplace à coefficients constants

$$
\bar{r}_{u v}=\frac{1}{n} \bar{r}_{u}+n \bar{r}_{v}
$$

dont les deux invariants sont égaux a l'unité.

11. Considérons enfin les surfaces Mayer-Gheorghiu, caractérisées par la propriété que l'invariant $K / d^{4}$ est constant seuelement le long d'une famille d'asymptotiques. Ces surfaces sont définies par le système 


$$
\begin{aligned}
r_{u u} & =\left(1+\frac{h_{u}}{h}\right) r_{u}+h\left[h-(L h)_{u v}\right] r_{v}, \\
r_{u v} & =r_{v}+h r \\
r_{v v} & =\frac{1}{h} r_{u}+\frac{h_{v}}{h} r_{v} .
\end{aligned}
$$

En effectuant les mêmes calculs nous trouvons les deux équations de Laplace

$$
\begin{aligned}
& \bar{r}_{u v}=\frac{\mu h}{\lambda}\left[h-(L h)_{u v}\right] \bar{r}_{u}+\left(1+\frac{\mu_{u}}{\mu}\right) \bar{r}_{v}, \\
& \bar{r}_{v u}=\left(\frac{h_{v}}{h}+\frac{\lambda_{v}}{\lambda}\right) \bar{r}_{u}+\frac{\lambda}{\mu h} \bar{r}_{v},
\end{aligned}
$$

qui sont aussi équivalentes. Pour les valeurs constantes de $h$ on peut déterminer encore des couples de deux surfaces parallèles, mais les calculs seraient plus longs.

\section{BibliogRAPHIE}

1. K. M. Peterson, Ob otnochenia i srodsvah mejdou crivemi povernostemi, Matematiceskir Sbornik (a. 1866).

2. - Sur les relations et les affinités entre les surfaces courbes, Annales de la Faculté des Sciences de l'Université de Toulouse (a.1905).

3. C. Guichard, Théorie des réseaux, Mémorial des Sciences Mathématiques.

4. M. Haimovici et I. Popa, La correspondance par plans tangentes parallèles, Annales Scientifiques de l'Université de Jassy vol. 18 (a.1933).

5. A. Myller, Curbe şi suprafete paralele in sens larg, Stud. Cerc. Sc. Iaşi (a.1954).

6. O. Mayer, Géométrie centro-affine différentielle des surfaces, Annales Scientifiques de l'Université de Jassy, vol. 21 (a.1935).

7. G. T. Gheorghiu, Sur une classe de surfaces, Mathematica (a.1942).

Timisoara, Roumania 